\title{
СИСТЕМНЫЕ И ЖАНРОВЫЕ ХАРАКТЕРИСТИКИ АНГЛОЯЗЬЧНОГО ДИСКУРСА ИНФОРМАЦИОННЫХ ТЕХНОЛОГИЙ
}

\section{SYSTEMIC AND GENRE CHARACTERISTICS OF THE ENGLISH-LANGUAGE DISCOURSE OF INFORMATION TECHNOLOGIES}

\section{N. Ayvazyan}

Summary: The article is devoted to the study and description of the systemic and genre characteristics of the modern English-language discourse of information technologies, verbalized in the non-fiction texts of the Internet resources. The relevance of this study is due to the intensive development of information technologies, generating changes in society, in the professional and scientific fields, contributing to the effective implementation of professional communication. The article describes the typical participants of the discourse of information technologies, its chronotope, goals, values, themes, strategies and genres. It was revealed that for the discourse of information technologies, actualizing professional knowledge in the field of information and computer communication, the integral characteristics are the popularization of scientific knowledge, promotion of professional value concepts, representation of certain professionally significant meanings, genre diversity.

Keywords: discourse of information technologies, concept, value, strategies, genres.
Айвазян Надежда Борисовна старший преподаватель, Кубанский государственный аграрный университет им. И.Т. Трубилина, г. Краснодар

n-ajvazyan@mail.ru

Аннотация: Настоящая статья посвящена изучению и описанию системных и жанровых характеристик современного англоязычного дискурса информационных технологий, воплощающегося в научно-популярных текстах Интернет-источников. Актуальность темы данного исследования обусловлена интенсивным развитием информационных технологий, порождающих в настоящее время изменения в обществе, в профессиональной и научной областях, способствующих эффективному осуществлению профессиональной коммуникации. В статье описаны типовые участники дискурса информационных технологий, хронотоп, цели, ценности, тематика, стратегии и жанры. Выявлено, что для дискурса информационных технологий, актуализирующего профессиональные знания в сфере информационно-компьютерной коммуникации, неотъемлемыми характеристиками являются популяризация научных знаний, продвижение профессиональных ценностных концептов, репрезентация определенных профессионально значимых смыслов, жанровое разнообразие.

Ключевые слова: дискурс информационных технологий, концепты, ценности, стратегии, жанры.
$\mathrm{B}$ ыявление характерных особенностей коммуникативного взаимодействия в информационном пространстве, обусловленное интенсивным развитием информационных технологий, порождающих в настоящее время изменения в обществе, в профессиональной и научной областях, определяет актуальность исследования профессиональной направленности англоязычного научно-популярного дискурса, посвященного информационным технологиям (ИТ).

Англоязычный дискурс информационных технологий является инструментом расширения информационнокоммуникативного пространства личности для индивидуально значимой и социальной активности, представляет собой сложную коммуникативную практику, оказывающую влияние на способы интерпретации информационных технологий обществом, репрезентируемую в текстах тематического характера об информационных технологиях в научно-популярных Интернет-изданиях, учебной литературе и направленных на адресата-непрофессионала [2; 4; 7].

Ученые, рассматривая дискурс информационных технологий как отдельную коммуникативную сферу, основываются на такой характерной особенности данного вида коммуникации, как единство предметной области и тематической организации текстов об ИТ, что способствует эффективному ведению диалога профессионалов и пользователей данных технологий [11]. Необходимо отметить, что особое внимание уделяется такой особенности текстов дискурса информационных технологий, как сочетание общих и профессиональных жанров, где экстралингвистические факторы, а именно привлечение внимания адресата визуальными средствами, играют важную роль [12].

Основываясь на прагматических категориях описания институциональных типов дискурса В.И. Карасика [8], охарактеризуем типовых участников дискурса информационных технологий, его хронотоп, цели, ценности, тематику, стратегии и жанры.

Исследуемый тип речевого взаимодействия относится, по мнению ученых, к массовой коммуникации, на основании того, что репрезентируемые в публици- 
стических Интернет-изданиях англоязычные тексты об ИТ создаются специалистами в области информатики, программирования, производства и разработки программного обеспечения, а участниками данного вида коммуникации может стать любой пользователь, как профессионал, так и непрофессионал, не принадлежащий к данной сфере профессионального общения [10].

Хронотоп изучаемого дискурса информационных технологий носит двойственный характер, поскольку участников данного вида коммуникации, находящихся одновременно в двух пространственно-временных позициях: виртуальной и реальной [6], объединяет виртуальное пространство, создаваемое сетью Интернет, при этом временная составляющая носит асинхронный характер. Необходимо отметить, что реальное пространство участников дискурса информационных технологий может отличаться, что обусловлено местом нахождения коммуникантов (служебные помещения, конференции, пункты Интернет-коммуникации, внеслужебная обстановка в разных концах земного шара) [6; 12].

К целям исследуемого дискурса относятся поиск и отбор информации для использования в производственном процессе и создания информативно-содержательных текстов, способствующих максимально эффективному восприятию, привлечению внимания адресата к описываемым продуктам и услугам, что обусловливает постоянное совершенствование подготовки кадров и развитие навыков специалистов и пользователей-непрофессионалов в области информационных технологий [12]. Необходимо подчеркнуть, что специфика текстов об ИТ обусловливает возможность рассмотрения дискурса информационных технологий в рамках разновидности научного стиля, выделяя популяризацию достоверных научных знаний, детерминированную логичностью изложения, доказательством истинности и ложности различных положений в качестве основной цели [1, с. 60; 13, с. 43].

Отметим, что исследователи рассматривают профессиональные тексты об ИТ как влияющий на адресата и формирующий характерные способы восприятия той или иной технологии инструмент создания и распространения системы ценностей, являющихся результатом когнитивно-языковой деятельности человека и фактором воздействия на формирование и распространение определенных смыслов и установок в обществе [3; 14]. А.Ю. Багиян, исследуя ценностную сферу научно-популярного дискурса, посвященного использованию ИТ, выявляет ценностные концепты аксиологической системы в сфере дискурса информационных технологий, актуализирующие определенные профессионально значимые смыслы (CAREER (KAPbEPA), CONNECTION (CВЯЗЬ), DATA (ДАННЫЕ), DOMINATION (ДОМИНИРОВАНИЕ), ENTERTAINMENT (РАЗВЛЕЧЕНИЕ), EVOLUTION (РАЗВИТИЕ),
FAMЕ (СЛАВА), FEEDВАСК (ОБРАТНАЯ СВЯЗЬ), INFLUENCE (ВЛИЯНИЕ), МОNЕY (ДЕНЬГИ), РRIVАСУ (ПРИВАТНОСТЬ), SECURITY (БЕЗОПАСНОСТЬ)) [2]. В исследовании Т.А. Ширяевой подчеркивается важная роль языка в актуализации ценностей профессионального дискурса, которые воплощаются в концептах, определяющих релевантную информацию об ИТ, и классифицируются по семантическому признаку: RELEVANCE (APPROVAL, AUTHENTICITY, ENTERTAINMENT, FAME, INFLUENCE, POPULARITY), RELATIONSHIP (ACCESSIBILITY, CONNECTION, FEEDBACK, RELATABILITY), PROFESSION (CAREER, COMPETITIVENESS, MONEY, PROMOTION, TIME) [14].

К основным стратегиям дискурса информационных технологий, актуализируемого в Интернет-текстах и характеризуемого смешением научно-популярного подстиля научного стиля и аналитического подстиля публицистики, которые обладают характерными для обоих типов особенностями (информирование и воздействие на массового адресата, экспрессивность и доступность изложения) [2], относятся следующие: организующая стратегия (формулировка проблемной ситуации и выделение предмета изучения); объясняющая стратегия (изложение полученных результатов исследования в приемлемой как для специалистов, так и неспециалистов форме); содействующая стратегия (определение области практического применения полученных результатов); оценивающая стратегия (экспертная оценка проведенного исследования) [8; 12].

Необходимо отметить, что стратегии изучаемого дискурса информационных технологий детерминируются жанровой принадлежностью текстов научно-популярных Интернет-изданий, охватывающих вопросы производства и разработки программного обеспечения, инструкции использования современных ИТ. К жанрам строгой профессиональной направленности, характеризуемым терминологической насыщенностью, научностью изложения, инструктивным характером сообщений, относятся гарантии, рекомендации, спецификации, пользовательские инструкции. Жанрам, идентифицируемым субъектной оппозицией «профессионал - непрофессионал» и рассматриваемым в качестве свободного обмена информацией технологической направленности, соответствуют рекламные рецензии, критические статьи, беседы с непрофессионалами, интервью со специалистами [4].

Проиллюстрируем жанровые особенности современного англоязычного дискурса информационных технологий, репрезентируемого в Интернет-текстах научно-популярных изданий. Текстовый фрагмент (1), относящийся к жанру пошаговой инструкции, ориентирован на техническую поддержку пользователей компьютеров [5] и содержит информацию по установке настроек безопасности аккаунта Google. 
(1) To set up two-step verification, and access all of your other Google account security settings, head to the My Account page on the web. Click the Security tab on the left-hand side, then find the Signing in to Google heading. There, you can configure two-step verification and change your password if you need to. Whatever else you do to protect your account, the usual advice about choosing a strong password still applies. Make it at least 10 characters long; a mix of letters, numbers, and symbols; and ensure it's as difficult to guess as possible. Don't reuse passwords either: Choose a code that's specific to your Google account (so a data hack of a different account won't compromise your Google account as well) [16].

В данном дискурсивном отрезке актуализируются такие составляющие, как технологическая ориентированность (to set up two-step verification; you can configure two-step verification), терминологичность (Google account security settings) и инструктивный характер, репрезентируемый при помощи императивных конструкций (head to the My Account page on the web; click the Security tab; make it at least 10 characters long; choose a code), которые относятся к отличительным характеристикам данного жанра.

Процесс жанрообразования является довольно динамичным и гибким, вследствие замещения одного жанра другим, трансформацией и взаимопроникновением жанров, что обусловлено исторической подвижностью категории жанра [4, с. 318]. Так, в научно-публицистических текстах дискурса информационных технологий наблюдается феномен комбинирования жанров. В текстовом фрагменте (2) представлено описание технических характеристик и функционирования продукта (CHROMEBOOK), в котором совмещается жанр спецификации и жанр рекламной рецензии.

\section{(2) ACER CHROMEBOOK SPIN 713}

\section{The best Chromebook for most people SPECIFICATIONS \\ CPU: Up to Intel Core i7-10510U \\ Graphics: Up to Intel UHD Graphics RAM: 4GB / 8GB \\ Screen: 13.5-inch, $2256 \times 1504$ pixels \\ Storage: $64 G B / 128 G B / 256 G B$}

It comes with a high-resolution, $2265 \times 1504$ pixel 13.5inch screen that can be folded over and used in tent mode, as well as plenty of power under the hood - we're talking up to an Intel Core i5-10210U processor, 8GB of RAM and $128 \mathrm{~GB}$ of SSD storage. This should be able to handle all the Chrome tabs and Android apps you throw at it, and then some.

Other extras that we appreciate include an HDMI out port - not something you see very often on a Chromebook and the 3:2 aspect ratio on the display, which means you can fit much more on the screen vertically, compared with a 16:9 or even a 16:10 aspect ratio display. A polished and professional-looking Chromebook. [17]. В данном фрагменте репрезентировано техническое описание продукта (CPU, Graphics, Screen, Storage), и реализуются стратегии убеждения при помощи языковых элементов усиления высказывания (The best Chromebook for most people; other extras that we appreciate; which means you can fit much more on the screen) с целью оказания эмоционального воздействия на потребителя приобрести описываемый продукт.

Жанр рекламной рецензии, представленный в текстовом фрагменте (3), характеризуется использованием приема диалогизации, рассматриваемого как преобразование монолога в диалог, запрос на обратную связь [9, с. 207]. Данный прием актуализируется при помощи использования местоимений, обращений, императивных конструкций с целью, эмоционального воздействия, привлечения внимания адресата, повышения заинтересованности в предлагаемой продукции.

(3) If you also invest in a top-rated gaming mouse, a high quality mechanical gaming keyboard, and an audiophile-quality gaming headset, you can be sure that your gaming setup will deliver immersion and quality for many years to come.

We're not just interested in performance, though: there's no point blowing your budget on a gaming laptop that isn't going to go the distance. That's why we're focusing on manufacturers such as Dell, Razer, Asus, Acer, MSI, HP and Alienware: manufacturers with a proven track record in delivering gaming machines that deliver rock-solid reliability.

On that note of specific manufacturers, Dell is making some great laptops right now for gamers, so head on over to our best Dell laptops guide for the complete picture. [18]. Языковые средства эмоционального воздействия (high quality, deliver immersion and quality for many years to come, a proven track record, great laptops) в сочетания с приемом диалогизации, использованием обращений (If you also invest in a top-rated gaming mouse... you can be sure that) и императива (so head on over to our best Dell laptops guide for the complete picture) усиливают динамичность обращения к потребителям, воздействие на адресата, способствуют реализации коммуникативного намерения автора рекламной рецензии.

В следующем фрагменте (4) представлен жанр критической статьи, в котором излагается мнение эксперта о новом программном расширении Google Docs. Автор описывает новый метод выполнения действий, используемых при работе с документами Google, неосложнённым терминами языком для пользователей-непрофессионалов.

(4) It's a clever new system of next-level shortcuts you can 
add into the Google Docs website - shortcuts that go beyond the basic options already built into the service and give you a more Slack-like, command-line-driven method of performing advanced actions. I've been using'em myself for a couple days now, and lemme tell ya: They totally change the way you use Google Docs - for the better.

The shortcuts come courtesy of a tasty and relatively new little Chrome extension called gSweets. The way it works is simple: You just add the extension into your browser, and the next time you open up a document in Docs on your computer, you'll see a snazzy new gray prompt at the beginning of every new line. That prompt pretty much tells you everything you need to know. From anywhere in a document, you can now simply hit the slash key on your keyboard - just like you would in Slack - and you'll be greeted with a scrolling list of quick ' $\boldsymbol{n}$ ' simple commands you can type in next... [15]. Как видим, отличительной особенностью данного отрывка критической статьи является использование разговорных формул английского языка (lemme tell ya; You just add the extension; you'll see a snazzy new gray prompt; quick ' $n$ ' simple commands), актуализирующих воздействие на читателя, подчеркивая нестрогость данного жанра дискурса информационных технологий, который предполагает наличие информационного обмена между профессионалами и непрофессионалами. Автор текста дополняет характеристику нового расширения для работы с ярлыками документов собственной оценкой (I've been using 'em myself for a couple days now, and lemme tell ya; They totally change the way you use Google Docs - for the better), наличие которой является неотъемлемой составляющей представленного жанра изучаемого типа дискурса.

Таким образом, дискурс информационных технологий как особая коммуникативная сфера профессионального общения людей, связанных с технологической индустрией, объединяющая совокупность текстов научной тематики, репрезентируемых в научно-публицистических Интернет-журналах и направленных как на адресатов-профессионалов, так и непрофессионалов с целью успешного овладения информационными технологиями, обладает своеобразными системными и жанровыми характеристиками. Популяризация достоверных научных знаний, продвижение профессиональных ценностных концептов, репрезентация определенных профессионально значимых смыслов, жанровое разнообразие являются неотъемлемыми характеристиками дискурса информационных технологий.

\section{ЛИТЕРАТУРА}

1. Аликаев Р.С. Язык науки в парадигме современной лингвистики. Нальчик, 1999. 317 с.

2. Багиян А.Ю., Аракелова А.Р. Концептуальное воплощение ценностей и антиценностей в научно-популярном дискурсе сферы информационных технологий как способ актуализации профессиональной аксиологической картины мира // Научный диалог. 2019. № 10. С. 61-78. DOl: 10.24224/2227-1295-2019-10-61-78.

3. Багиян А.Ю., Ширяева Т.А., Аракелова А.Р. Лингвоаксиологические характеристики научно-популярного дискурса информационных технологий // Филологические науки. Научные доклады высшей школы. М.: Алмавест, 2019. № 4. С. 33-41.

4. Галичкина Е.Н. Типология жанров компьютерного дискурса (компьютерная коммуникация) // Вестник Московского государственного лингвистического университета. 2009. № 549. С. 317-327.

5. Галичкина Е.Н. Компьютерная коммуникация: лингвистический статус, знаковые средства, жанровое пространство: автореф. дисс. ... д-ра филол. наук: 10.02.19. Волгоград, 2012. $40 \mathrm{c}$.

6. Гриценко Л.М. Особенности виртуального дискурса // Молодой ученый. 2011. № 6 (29). Т. 2. С. 17-20.

7. Елагина Ю.С. Системные характеристики профессионального компьютерного дискурса. // Известия Самарского научного центра Российской академии наук. 2010. т. 12. № 5 (2). С. 502-507.

8. Карасик В.И. Языковой круг: личность, концепты, дискурс. Волгоград: Перемена, 2002. 477 с.

9. Моногарова А.Г. Современный электронный текст: жанровое многообразие и прагматический потенциал // Профессиональная коммуникация: актуальные вопросы лингвистики и методики. 2020. №13. С. 205-210.

10. Николова Д. Коммуникация в сфере информационных технологий и жаргон как одно из ее средств. // Litera scripta manent. Служение слову. 2015. Т. 2. C. $111-129$

11. Орлов Е.А. Профессиональный компьютерный дискурс как особая сфера общения специалистов // Гуманитарный вестник, 2013. вып. 3(5). [Электронный ресурс]. URL: http://hmbul.bmstu.ru/catalog/lang/ling/46.html (дата обращения 20.04.2021).

12. Руженцева Т.С. Профессиональный дискурс специалиста по информационным технологиям как основной механизм передачи информации // Гуманитарное образование в экономическом вузе: Материалы IV Международной научно-практической заочной интернет-конференции. Москва: Российский экономический университет имени Г.В. Плеханова. 2016. С. 354-359.

13. Чернявская В.Е. Интерпретация научного текста. М.: КомКнига, 2006. 128 с.

14. Shiryaeva T., Arakelova A., Golubovskaya E., Mekeko N. Shaping values with «YouTube freedoms»: linguistic representation and axiological charge of the popular science IT-discourse. Heliyon. 2019. T. 5. № 12. C. e02988.

15. Computerworld [Electronic Resource]. Mode of Access: https://www.computerworld.com/article/3603909/google-docs-shortcuts.html 
16. Popular Science [Electronic Resource]. Mode of Access: https://www.popsci.com/secure-your-google-account/

17. T3 [Electronic Resource]. Mode of Access: https://www.t3.com/features/best-chromebook

18. T3 [Electronic Resource]. Mode of Access: https://www.t3.com/features/best-gaming-laptop

( ) Айвазян Надежда Борисовна (n-ajvazyan@mail.ru).

Журнал «Современная наука: актуальные проблемы теории и практики»

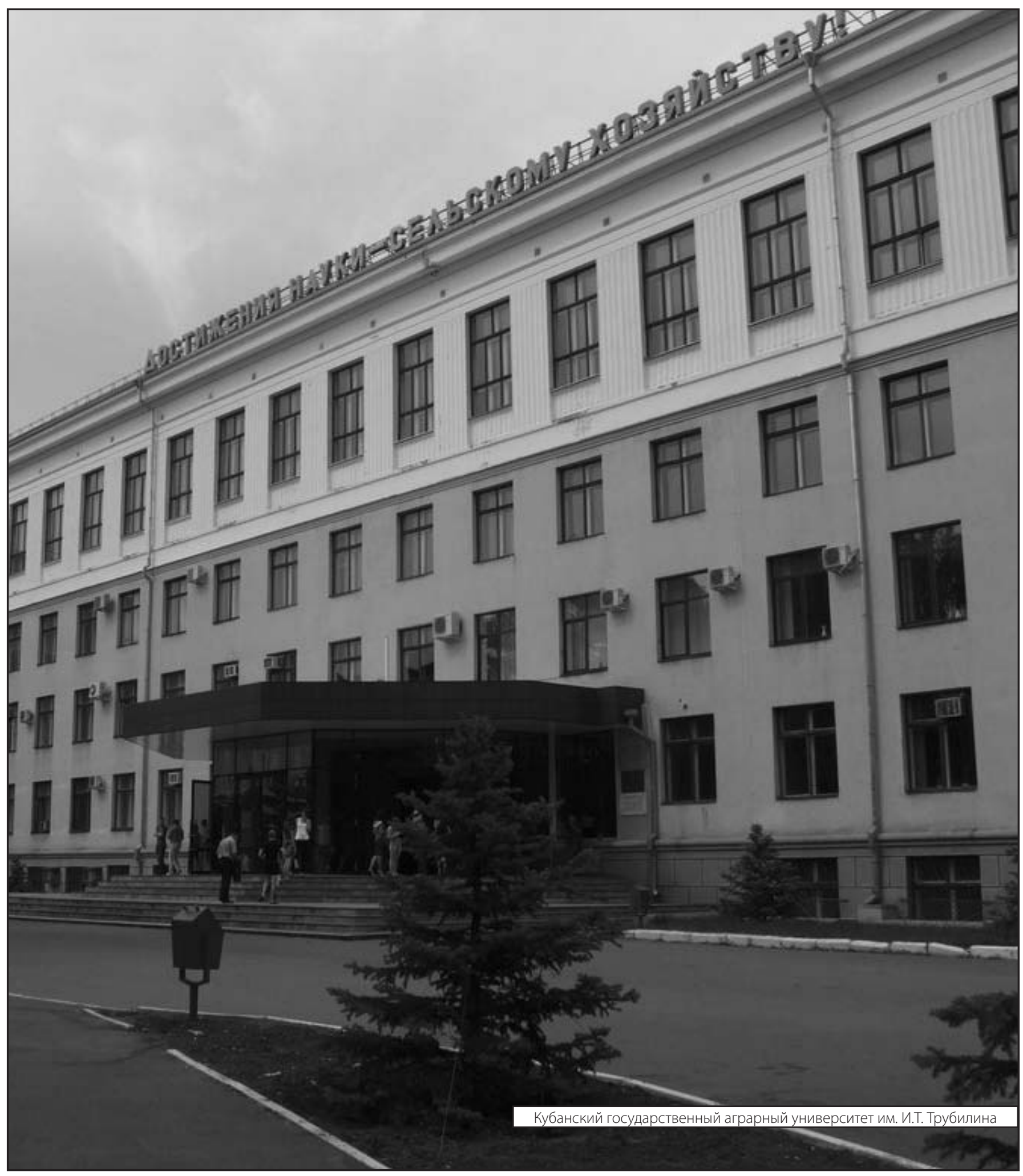

